\title{
Hand Gesture Alphabet Recognition for American Sign Language using Deep Learning
}

\author{
Krutika S. Kale;, Prof. Milind B. Waghmare
}

Department of Computer Science and Engineering, Government College of Engineering Amravati, India

\section{Article Info}

Volume 8, Issue 5

Page Number : 213-220

\section{Publication Issue :}

September-October-2021

\section{Article History}

Accepted : 20 Sep 2021

Published: 30 Sep 2021

\section{ABSTRACT}

Speech impairment limits a person's capacity to speak and communicate with others, forcing them to adopt other communication methods such as sign language. Sign language is not that widely used technique by the deaf. To solve this problem, we developed a powerful hand gesture detection tool that can easily monitor both dynamic and static hand motions with ease. Gesture recognition aims to translate sign language into voice or text for individuals who have a rudimentary comprehension of that, which will be a tremendous help in communication between deaf-mute and hearing people. We describe the design and implementation of an American Sign Language (ASL) fingerspelling translator based on spatial feature identification using a convolutional neural network.

Keywords : Sign Language Recognition, Deep learning, image processing, American sign Language, Hand gesture detection.

\section{INTRODUCTION}

Sign language is a type of interaction used by those who have difficulty hearing and speaking. People employ nonverbal communication such as signal language gestures to express their thoughts and emotions. Non-signers, on the other hand, find it extremely difficult to comprehend, which is why trained signal language translators are sought at various stages in scientific and criminal appointments, as well as academies. Over the last five years, there is an increasing demand for decoding services. Other methods, such as video decoding from afar using high-speed internet connections, had been introduced. As a result, they will provide an easy-touse signal language decoding service, that can be employed, but has a major drawback regarding internet connectivity and the appropriate gadget. Signal language recognition can be accomplished in a variety of ways, including glove-based completely popularity and vision-based totally popularity. A network of sensors is utilised to capture the actions of the fingers in a glove-based manner. The proposed device employs a non-invasive vision-based fully popularity technique. The vision-primarily based entirely popularity can be carried out in two ways: static popularity or dynamic popularity with the use of CNN. Convolution Neural Network, is a powerful 
learning algorithm which is utilized for cleaning and extracting capabilities from photographs.

The mouse and keyboard have played an important part in HCI for many years. Speech and gesture recognition systems garner a lot of attention. A gesture is a physical and emotional expression symbol. It consists of both hand and body motions Gestures are a way for a computer and a person to communicate. Traditional hardware-based methods for achieving human-computer interaction are vastly different from gesture recognition. The person's intention is detected using sign language, which recognises the actions of the parts of the body. Numerous academics have competed in recent years to enhance hand motion recognition technology Augmented reality, disabled and robot control and sign language interpreters are only a few of the uses for hand gesture detection. Gestures, like speaking, are a natural way for people to communicate with one another. They could be the most natural way of expressing yourself. This is due to the fact that infants transmit their emotions and desire to speak through hand movements.

The challenge of comprehending human motions can be solved via pattern recognition. If a computer can recognise human motion patterns, the required message may be recreated. It has been proven that static sign motions used to indicate alphabets and numerals may be detected. Here, this system has been extended to recognize alphabets and sentences in American sign language.

A number of different hand gestures have sprung significantly as a part of this. Speak language is the predominant mode of communication for the great majority of the population. Despite the existence of verbal communication, some people are not able to convey the message with majority. This group of persons can benefit from sign language. Face expressions, static hand signals, plus hand gestures have been used in sign language in order to communicate in a similar way to that of verbal communication does. Sign languages can be of many types.

\section{RELATED WORK}

The literature review done sheds light on the many approaches for hand gesture recognition that may be adopted and applied. It also aids in comprehending the benefits and drawbacks connected with certain approaches. The camera module in this determines which cameras and markers are available for use. The detection module is in charge of picture preprocessing and feature extraction.

Data gloves, hand belts, and cameras are some of the most prevalent techniques of collecting human input that have been seen. The gesture recognition [1] and [2] approaches employ data gloves to extract input. To read hand motions, a hand belt containing a gyroscope, accelerometer, and Bluetooth was utilized [3] [4]. To collect both color and depth information, the authors [5] utilized a Senz3D Camera. The SAD (Sum of Absolute Differences) method was used to compare the left and right pictures. $\mathrm{m}$. The Theo Pavildis Algorithm was used to identify the contours in [6], which visits just the border pixels. The computational expenses are reduced with this approach.

[7], [8], and [9] are cost-effective models that have been implemented. Simple web cams are used in their systems.[10] Uses both color and depth pictures, a recognition system based on 24 static American Sign Language (ASL) alphabet signs was created. Gabor filters are a type of filter used to extract characteristics at various sizes and for multiclass classification the classifier was a random forest. In the leave-one-out trial, they had a recognition rate of 49 percent. They also created the American Fingerspelling dataset. The most often used benchmarked dataset is this one. When it comes to sign language research, this is a great place to start. The hand palm contour was chosen as the largest contour in [8] [11] [13], followed by the Polygonal approximation was used to simplify the contour. Individual objects are grouped together in a classification process based on their similarity. 
[12] Two unique CNN-based models that can detect 24 static ASL signals were built. They separated one dataset into colur pictures and the other into a mix of colour and depth images. They achieved 86.52 percent and 85.88 percent accuracy for each of their models, and they contrasted their results with these two sets of datasets utilising transfer learning with pretrained models such as VGG 19, VGG 16, and others. The majority of methods allow for the extraction of a hand area.

The method [14] recognizes 25 hand poses using a Euclidean distance-based classifier. In [15] and [16], the Support Vector Machine (SVM) classifier was employed. We depart from the norm alternative conventional methods for gesture recognition that do not need the use of any hand markers, such as gloves. To keep the system cost-effective, we used a webcam built into the laptop rather than purchasing extra cameras. As a result, our system has applications in everyday life.

\section{PROPOSED METHODOLOGY}

There are two methods for recognising sign language: glove-based recognition and vision-based recognition. This system employs a vision-based recognition mechanism that is non-invasive. There are two methods for achieving vision-based recognition. The entry point file will be manually run on any modern browser, notably the most recent updated Chrome browser classifier.

This system includes a camera unit for recording and generating, as well as training for hearing and speech challenged people's gestures. The pictures scanned from the raw videos are fed into the system with the correct environmental configuration. To ensure that all of the movies are similar in size, the picture frames are adjusted. Feature extraction and classification are done using Convolution Neural Networks. To access web camera, the programme will display a notification. We can read American sign language using camera and write the character on a screen after allowing webcam access. Using unique hand movements, any two words can be separated by space. After you finish writing on the screen, press the convert to Audio button to convert the text to audio.

The planned work flow is broken into three parts:

1. Compiling the data

2. Use the captured dataset to train a CNN.

3. Data forecasting

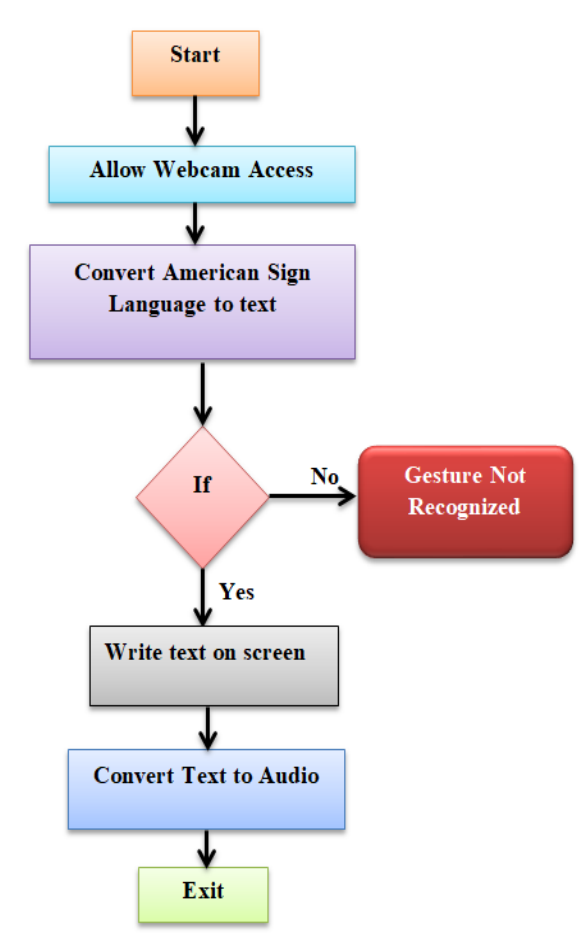

Fig. 1. Flow of Proposed Work

The working process of the model is executed by the flow given below,

Step 1: In the first step, we have to upload data.

Step 2: Data may be trained using CNN by following the steps of the CNN algorithm. A loss graph and model summary will be created when training is completed.

Step 1: set your neural network options

Step 2: initialize your neural network

Step 3: normalize data and train the model

Step 4: train the model

Step 5: use the trained model 
Step 6: make a classification

Step 3: For testing upload testing data.

Step 4: Test pretrain model using testing data, testing will return confusion matrix, accuracy, precision, and recall of pretrain model. Following $\mathrm{CNN}$ algorithm steps will be done in the testing model.

Step 1: Load pretrained model, the weights, and the metadata.

Step 2: define a function to handle the results of your classification

Step 5: In the output model single prediction will be done. Following CNN algorithm steps will be done in the output model.

Step 1: Load pretrained model, the weights, and the metadata.

Step 2: define a function to handle the results of your classification.

\section{RESULT}

\section{A. Result}

The performance of the proposed methodology with a different number of layers and epoch has been successfully evaluated and the output for each layer is as shown below.

\section{1 Layer and 180 Epoch}

- Algorithm Used: CNN

- Number of layers: 1

- Activation Function: SoftMax

- No of epoch: 180

- Graph for Loss:

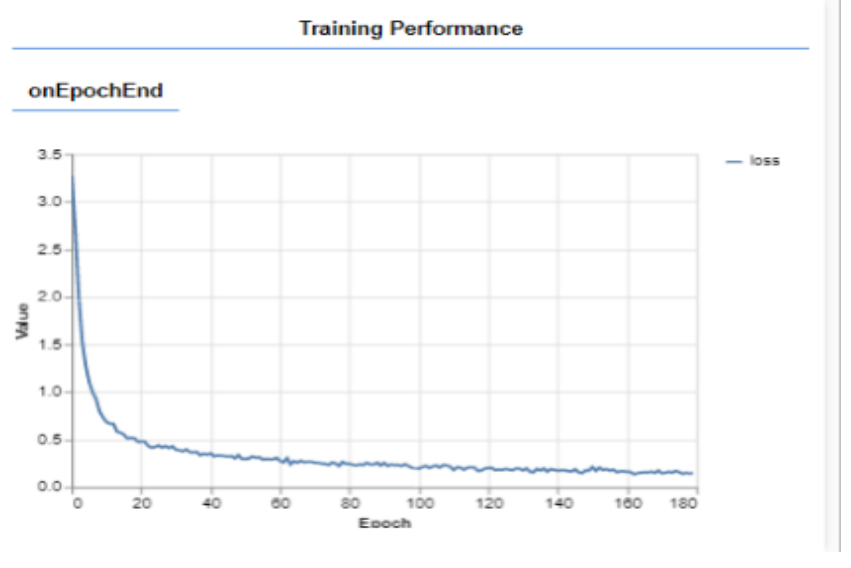

- Model Summary:

\begin{tabular}{llll}
\multicolumn{3}{c}{ Model Summary } & \\
\hline Layer Name & Output Shape & \# Of Params & Trainable \\
\hline conv2d_Conv2D1 & [batch,60,60,8] & 808 & true \\
\hline max_pooling2d_MaxPooling2D1 & [batch, 30,30,8] & 0 & true \\
\hline flatten_Flatten1 & [batch,7200] & 0 & true \\
\hline dense_Dense1 & [batch,27] & 194,427 & true \\
\hline
\end{tabular}

- Dataset Information: For testing used dataset from Kaggle

- Number of Images used for training: used a custom based dataset which will be taken with the help of video.

- Number of Images used for testing $: 20 * 27$ (classes) $=540$

- Project Accuracy: 0.96

- Precision: 0.96

- Recall: 1

- F-1 Score: 0.98

- Confusion matrix:

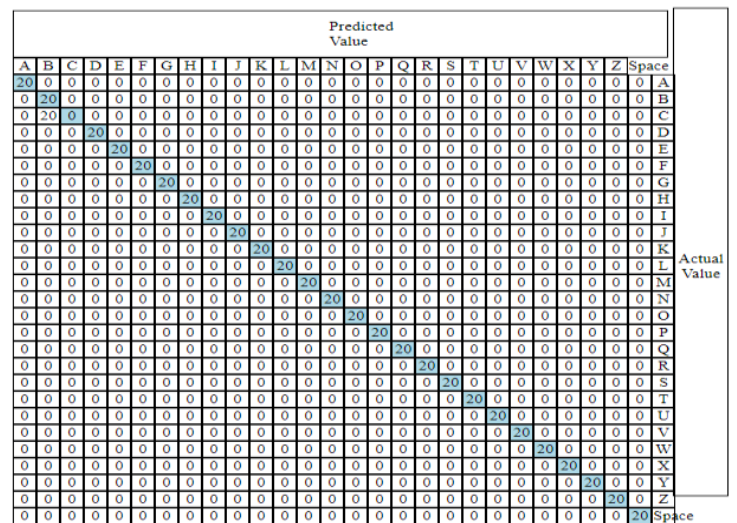




\section{2 Layers and 100 Epoch}

- Algorithm Used: CNN

- Number of layers: 2

- Activation Function: SoftMax

- No of epoch: 100

- Graph for Loss:

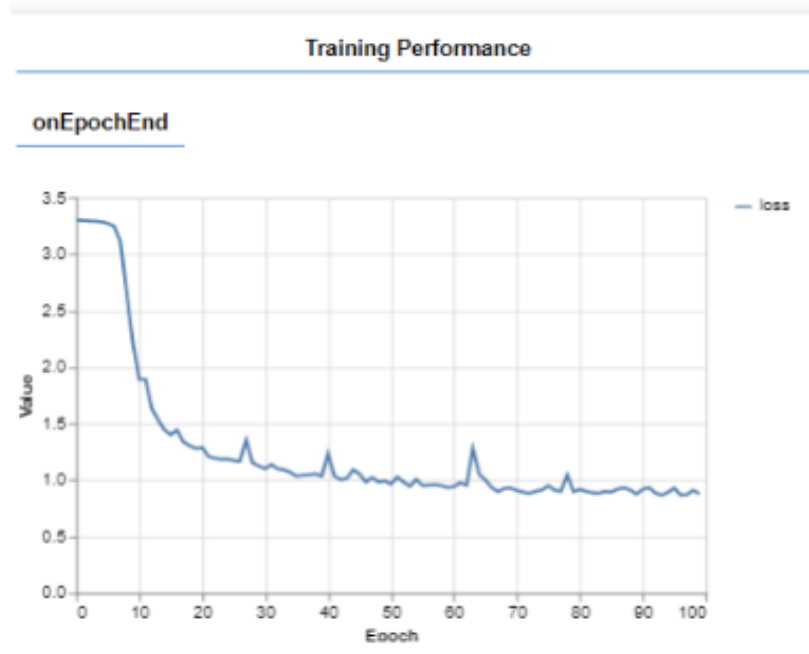

- Model Summary:

\begin{tabular}{llll}
\multicolumn{5}{c}{ Model Summary } & & \\
\hline Layer Name & Output Shape & \# Of Params & Trainable \\
\hline conv2d_Conv2D1 & {$[$ batch,60,60,8] } & 808 & true \\
\hline max_pooling2d_MaxPooling2D1 & {$[$ batch, 30,30,8] } & 0 & true \\
\hline conv2d_Conv2D2 & {$[$ batch,26,26,8] } & 1,608 & true \\
\hline max_pooling2d_MaxPooling2D2 & {$[$ batch, 13,13,8] } & 0 & true \\
\hline flatten_Flatten1 & {$[$ batch,1352] } & 0 & true \\
\hline dense_Dense1 & {$[$ batch,27] } & 36,531 & true \\
\hline
\end{tabular}

- Dataset Information: For testing used dataset from Kaggle

- Number of Images used for training: used a custom based dataset which will be taken with the help of video.

- Number of Images used for testing: $20 * 27$ (classes) $=540$

- Project Accuracy: 0.94

- Precision: 0.94

- Recall: 1

- F-1 Score: 0.97

- Confusion matrix:

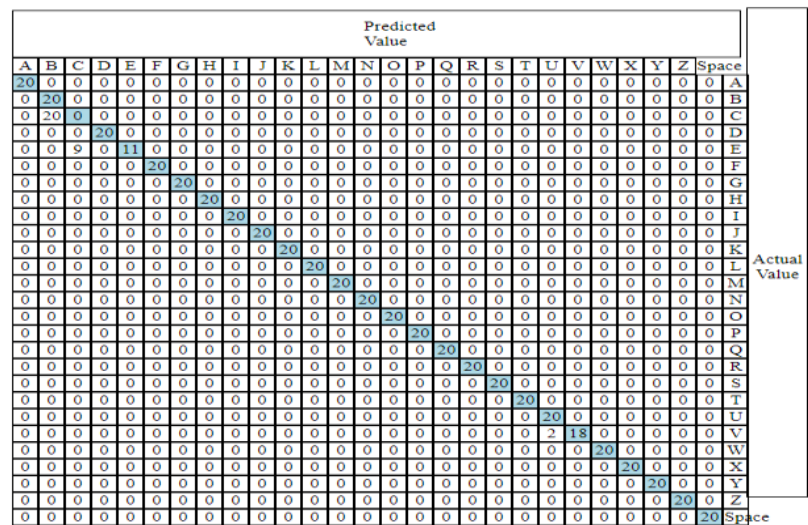

\section{3 Layers and 50 Epoch}

- Algorithm Used: CNN

- Number of layers: 3

- Activation Function: SoftMax

- No of epoch: 50

- Graph for Loss:

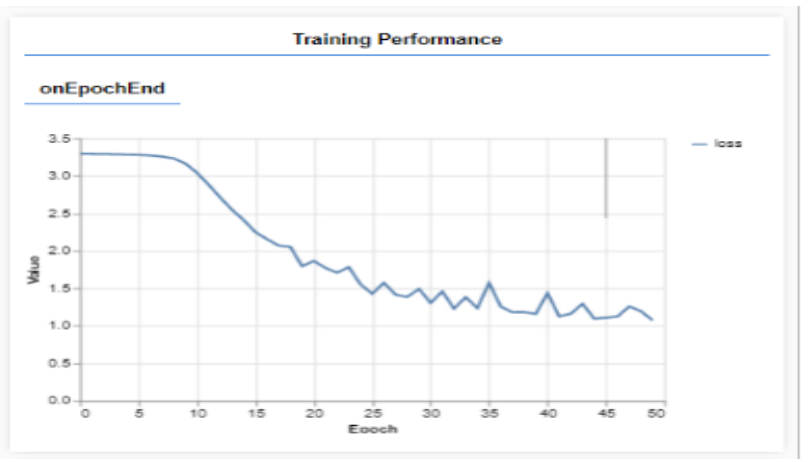

- Model Summary:

\begin{tabular}{|c|c|c|c|}
\hline \multicolumn{4}{|c|}{ Model Summary } \\
\hline Layer Name & Output Shape & \# Of Params & Trainable \\
\hline conv2d_Conv2D1 & [batch, $60,60,8]$ & 808 & true \\
\hline max_pooling2d_MaxPooling2D1 & [batch, $30,30,8]$ & 0 & true \\
\hline conv2d_Conv2D2 & [batch, $26,26,8]$ & 1,608 & true \\
\hline max_pooling2d_MaxPoolling2D2 & [batch, 13,13,8] & 0 & true \\
\hline conv2d_Conv2D3 & [batch, 9,9,8] & 1,608 & true \\
\hline max_pooling2d_MaxPooling2D3 & [batch, $4,4,8]$ & 0 & true \\
\hline flatten_Flatten 1 & [batch, 128] & 0 & true \\
\hline dense_Dense1 & [batch,27] & 3.483 & true \\
\hline
\end{tabular}

- Dataset Information: For testing used dataset from Kaggle

- Number of Images used for training: used a custom based dataset which will be taken with the help of video.

- Number of Images used for testing: $20 * 27$ (classes) $=540$ 
- Project Accuracy :0.83

- Precision:0.89

- Recall: 0.93

- F-1 Score: 0.91

- Confusion matrix:

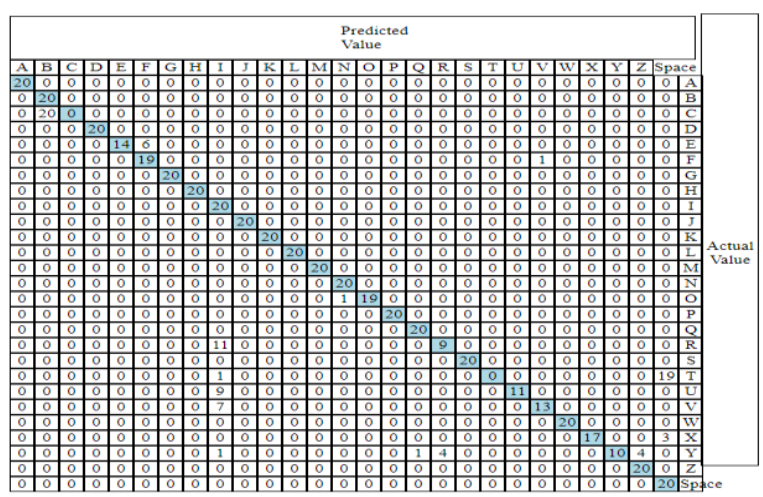

B. Performance Analysis

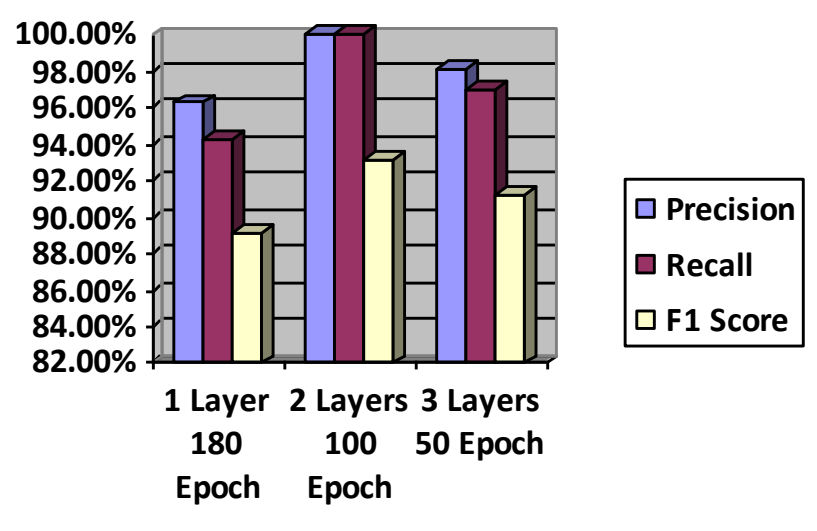

Fig. 2. Precision, Recall and F1 score for three different layers

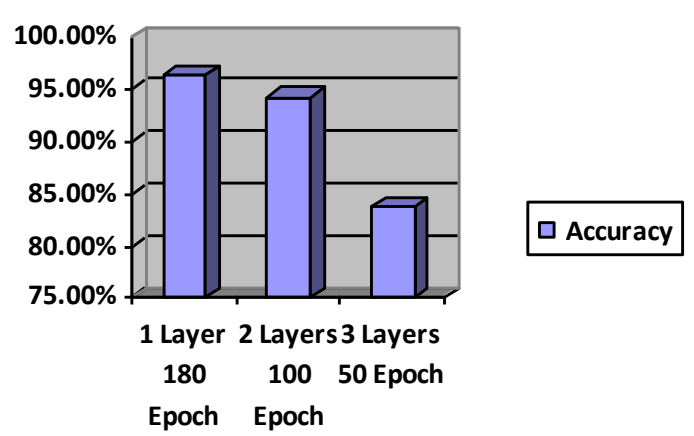

Fig. 3. Accuracy of three different layers V. CONCLUSION
The vision-based method for the recognition of hand gestures for American sign language is presented in this paper. The characteristics retrieved from the sign picture are utilised to train a sign recognition feed forward neural network. The device can identify 27 hand gestures, including the letters $\mathrm{A}$ to $\mathrm{Z}$ and a unique symbol for space. This method is a novel approach to aiding people who have difficulty communicating due to speech or voice problems. The vision is to develop a social application which will make it simpler for deaf and mute people to communicate by utilising image processing techniques. Because it employs an image-based approach, it may be started as an application in any basic system and has a near-zero cost. Here with the help of CNN algorithm using 1 layer 180 Epoch we get highest accuracy which is $96.29 \%$.

\section{REFERENCES}

[1]. Granit Luzhnica, Elizabeth Lex, Viktoria Pammer. A Sliding Window Approach to Natural Hand Gesture Recognition using a Custom Data Glove. In: 3D User Interfaces (3DUI); 2016 IEEE Symposium on ; $2016 \mathrm{Mar}$ 19 ; New York : IEEE; 2016 ; p.81-90.

[2]. ]Ji-Hwan Kim,Nguyen Duc Thang,Tae-Seong Kim. 3-D hand Motion Tracking and Gesture Recognition Using a Data Glove. In; Industrial Electronics; 2009 IEEE International Symposium on ; 2009 July 5; New York : IEEE;2009; p.1013-1018.

[3]. Hung CH, Bai YW, Wu HY. Home outlet and LED array lamp controlled by a smartphone with a hand gesture recognition. In: Consumer Electronics (ICCE); 2016 IEEE International Conference on ; 2016 Jan 7; New York : IEEE;2016; p.5-6.

[4]. Hung CH, Bai YW, Wu HY. Home appliance control by a hand gesture recognition belt in LED array lamp case. In: Consumer Electronics (GCCE); 2015 IEEE 4th Global 
Conference on ; 2015 Oct 27; New York : IEEE;2015; p. 599-600

[5]. She Y, Wang Q Jia Y, Gu T, He Q Yang B. A real-time hand gesture recognition approach based on motion features of feature points. In: Computational Science and Engineering (CSE); 2014 IEEE 17th International Conference on; 2014 Dec 19; New York: IEEE;2014;p.1096- 1102.

[6]. Lee DH, Hong KS. A Hand gesture recognition system based on difference image entropy. In: Advanced Information Management and Service (IMS), 2010 6th International Conference on; 2010 Nov 30; Seoul; New York: IEEE; 2010 ; p. 410-413.

[7]. Tsai TH, Huang CC, Zhang KL. Embedded virtual mouse system by using hand gesture recognition. In: Consumer Electronics-Taiwan (ICCE-TW); 2015 IEEE International Conference on; 2015 Jun 6; Taiwan, Taipei; New York: IEEE; 2015; p. 352-353.

[8]. Hussain I, Talukdar AK, Sarma KK. Hand gesture recognition system with real-time palm tracking. In: India Conference (INDICON);2014 Annual IEEE ;2014 Dec 11; India, Pune; New York: IEEE; 2014; p. 1-6.

[9]. Huong TN, Huu TV, Le Xuan T. Static hand gesture recognition for vietnamese sign language (VSL) using principle components analysis. In: Communications, Management and Telecommunications (ComManTel); 2015 International Conference on; 2015 Dec 28; p. 138-141.

[10]. Pugeault, N., Bowden, R., 'Spelling it out: Real-time asl fingerspelling recognition'. In: Computer Vision Workshops (ICCV Workshops), 2011 IEEE International Conference on. IEEE, pp. 1114-1119

[11]. Chen WL, Wu CH, Lin CH. Depth-based hand gesture recognition using hand movements and defects. In: Next-Generation Electronics (ISNE); 2015 International
Symposium on; 2015 May 4 ; Taiwan, Taipei ; New York : IEEE;2015 ;p. 1-4.

[12]. Pias Paul, Moh. Anwar-Ul-Azim Bhuiya, Md. Ayat Ullah, Molla Nazmus Saqib, Nabeel Mohammed, and Sifat Momen. 'A Modern Approach for Sign Language Interpretation Using Convolutional Neural Network', 16th Pacific Rim International Conference on Artificial Intelligence Cuvu, Yanuca Island, Fiji, August 26-30, 2019, Lecture Notes in Artificial Intelligence, Proceedings, Part III .

[13]. Suriya R, Vijayachamundeeswari V. A survey on hand gesture recognition for simple mouse control. In: Information Communication and Embedded Systems (ICICES); 2014 International Conference on; 2014 Feb 27; India, Chennai; New York : IEEE;2014;p. 1-5.

[14]. Luzhnica G, Simon J, Lex E, Pammer V. A sliding window approach to natural hand gesture recognition using a custom data glove. In :3D User Interfaces (3DUI); 2016 IEEE Symposium on; 2016 Mar 19; Greenville, SC ; New York : IEEE;2016 ;p. 81-90.

[15]. Chen Y, Ding Z, Chen YL, Wu X. Rapid recognition of dynamic hand gestures using leap motion. In: Information and Automation; 2015 IEEE International Conference on; 2015 Aug 8; New York : IEEE;2015 ;p. 1419-1424.

[16]. Chen Y, Luo B, Chen YL, Liang G, Wu X. A real-time dynamic hand gesture recognition system using kinect sensor. In: Robotics and Biomimetics (ROBIO); 2015 IEEE International Conference on ; 2015 Dec 6; New York : IEEE;2015; p. 2026-2030.

[17]. Walaa Aly, Saleh Aly, and Sultan Almotairi, User-Independent American Sign Language Alphabet Recognition Based on Depth Image and PCANet Features, 2020 IEEE Access Digital Object Identifier 10.1109/ACCESS.2019.2938829.

[18]. Rajesh George Rajan, Dr.M.Judith Leo, American Sign Language Alphabets 
Recognition using Hand Crafted and Deep Learning Features, 2020 Proceedings of the Fifth International Conference on Inventive Computation Technologies.

[19]. W. Tao, M. C. Leu, and Z. Yin, "American sign language alphabet recognition using convolutional neural networks with multiview augmentation and inference fusion,” Eng. Appl. Artif. Intell., vol. 76, pp. 202-213, Nov. 2018.

[20]. T.-Y. , Lo, L.-Y., Yeh, C.-W. , Li, J.-W. , Liu, H.-T. , \& Hu, M.C., Real-time sign language recognition in complex background scene based on a hierarchical clustering classification method, In Proceedings of the IEEE second international conference on multimedia big data (BIGMM) ,64-67, IEEE (2016).

[21]. Krutika S. Kale, Prof. Milind B. Waghmare, "Review on Hands Gestures Using American Sign Languages", International Journal of Scientific Research in Computer Science, Engineering and Information Technology (IJSRCSEIT), ISSN : 2456-3307, Volume 7 Issue 3, pp. 228-232, May-June 2021. Available at doi : https://doi.org/10.32628/CSEIT217361 Journal URL : https://ijsrcseit.com/CSEIT217361

[22]. Krutika S. Kale, Prof. Milind B. Waghmare, "Sign Language Recognition using CNN and ASL Dataset", International Journal for Research in Engineering Application \& Management (IJREAM) ISSN : 2454-9150 Vol-07, Issue-03, JUNE 2021.

\section{Cite this article as :}

Krutika S. Kale, Milind B. Waghmare, "Hand Gesture Alphabet Recognition for American Sign Language using Deep Learning", International Journal of Scientific Research in Science, Engineering and Technology (IJSRSET), Online ISSN : 2394-4099, Print ISSN : 2395-1990, Volume 8 Issue 5, pp. 213-220, September-October 2021. Available at doi : https://doi.org/10.32628/IJSRSET218521 Journal URL : https://ijsrset.com/IJSRSET218521 